INVESTIGACIÓN

https://doi.org/10.15198/seeci.2018.46.35-51

Recibido: 15/08/2017 --- Aceptado: 14/10/2017 --- Publicado: 15/07/2018

\title{
DE LA COLABORACIÓN FUERA DE LÍNEA A LA LÍNEA: ENFOQUE TEÓRICO SOBRE LOS ESTUDIOS DE COOPERACIÓN HUMANA Y LA ECONOMÍA COLABORATIVA
}

\section{FROM OFFLINE TO ONLINE COLLABORATION: THEORETICAL APPROACH ON HUMAN COOPERATION STUDIES AND THE SHARING ECONOMY}

Esther Martos Carrión: Universidad Charles de Praga. República Checa. sharing.economy.prague@gmail.com

\section{RESUMEN}

Desde mediados de los años 60, se han publicado varios enfoques científicos basados en experimentos sociales, técnicas de observación y metodologías etnográficas que buscan explorar y comprender comportamientos humanos (fuera de línea) en términos de cooperación y construcción comunitaria, así como acciones racionales egoístas. Sin embargo, en la última década, un sistema socio-económico innovador, impulsado por la tecnología y totalmente basado en plataformas en línea, la economía colaborativa, parece perturbar las formas establecidas de colaboración. Este estudio considera que, dada la novedad de la economía colaborativa, todavía faltan estudios empíricos que intenten comparar y conectar formas de actuar en línea y fuera de línea. Por lo tanto, el objetivo principal de este documento es comprender hasta qué punto las teorías relevantes sobre la cooperación humana, formuladas a partir de 1965 en adelante, podrían ser adecuadas para explicar el comportamiento colaborativo de la economía colaborativa impulsada digitalmente.

PALABRAS ClAVE: Economía compartida; consumo colaborativo; bienes comunes; Internet; las acciones racionalistas egoístas; cooperación

\begin{abstract}
Since the mid-60's, several scientific approaches based on social experiments, observational techniques and ethnographic methodologies have been issued seeking to explore and understand (offline) human behaviours in terms of cooperation and community building as well as selfish rational actions. However, over the last decade, an innovative socio-economic system, technologically driven and entirely based on online platforms, the sharing economy appears to disrupt stablished manners of collaboration. This study considers that, given the novelty of the sharing economy, there is still a lack of empirical studies attempting to compare and connect both offline and online forms of acting together. Thus, the main goal of this paper is to understand to what extent relevant theories on human cooperation, formulated from
\end{abstract}

\footnotetext{
${ }^{1}$ Esther Martos Carrión: Facultad de Humanidades, Universidad Charles de Praga, Departamento de Sociología Histórica, República Checa.

sharing.economy.praque@gmail.com
} 
1965 onwards, might be suitable for explaining the collaborative behaviour of the digitally driven sharing economy.

KEYWORDS: Sharing Economy; Collaborative Consumption; the Commons; the Internet; Selfish Rational Actions; Cooperation.

\title{
DA COLABORAÇÃO ANALÔGICA À COLABORAÇÃO EM LINHA: APROXIMAÇÃO TEÓRICA DOS ESTUDOS DE COOPERAÇÃO HUMANA E ECONÔMIA COLABORATIVA
}

\begin{abstract}
RESUME
Desde meados dos anos 60, várias aproximações cientificas baseadas em experimentos sociais, técnicas de observação e metodologias etnográficas foram tentadas, buscando explorar e entender o comportamento humano (analógico) em términos de cooperação e construção de comunidades, assim como as reações racionais egoístas. Sem embargo, a última década, um inovador sistema sócio econômico, impulsado pela tecnologia e inteiramente baseado em plataformas em linha, a economia colaborativa, parece transtornar os padrões estabelecidos de cooperação. Este estudo considera que, dado ao novo aspecto da economia colaborativa, ainda há uma falta de estudos empíricos que busquem comparar e conectar os modos analógicos e em linha de atuar em conjunto. Por isso, o principal objetivo deste estudo é entender até que extremo as teorias relevantes sobre cooperação humana, formuladas desde 1965, podem ser adequadas para explicar o comportamento cooperativo de uma economia colaborativa impulsada digitalmente.
\end{abstract}

PALAVRAS ChAVE: Economia Compartilhada; Consumo Colaborativo; Comuns; Internet; Ações Racionais Egoístas, Cooperação

\section{How to cite the article}

Martos Carrión, E. (2018). De la colaboración fuera de línea a la línea: enfoque teórico sobre los estudios de cooperación humana y la economía colaborativa [From offline to online collaboration: theoretical approach on human cooperation studies and the sharing economy] Revista de Comunicación de la SEECI, 46, 35-51. doi: http://doi.org/10.15198/seeci.2018.46.35-51. Recuperado de http://www.seeci.net/revista/index.php/seeci/article/view/501

\section{INTRODUCCIÓN Y PROPÓSITO DEL ESTUDIO}

La economía colaborativa, también conocida como consumo colaborativo (Felson y Spaeth, 1978; Algar, 2007; Botsman y Rogers, 2010), ha alcanzado un considerable nivel de popularidad en los países desarrollados. San Francisco, París, Londres, Amsterdam y Nueva York son capitales de la economía colaborativa en la que plataformas pioneras como Uber y AirBnB están perturbando progresivamente los mercados tradicionales. Este sistema socioeconómico intenta principalmente capacitar a los pares para compartir, intercambiar, intercambiar o alquilar sus bienes infrautilizados dentro de las comunidades en línea (Matofska, 2016). Los expertos en 
economía colaborativa (por ejemplo, Gansky, 2010; Owyang, 2013; Rifkin, 2014) afirman que el rendimiento óptimo de este modelo de consumo colaborativo basado en plataforma traerá múltiples beneficios a las sociedades. Por ejemplo, (1) está sostenido fundamentalmente por redes descentralizadas 0 distribuidas, contrariamente a las economías capitalistas que se configuran bajo estructuras piramidales y centralizadas (Botsman y Rogers, 2010). La igual distribución del poder y el control entre todos los miembros de la misma comunidad contribuye a aumentar los niveles de cooperación y confianza (Benkler, 2004). Como tal, los proveedores y consumidores de negocios de economía compartida tendrían más probabilidades de confiar el uno al otro dado que las plataformas se basan en relaciones de igual a igual (p2p), es decir, ambas partes organizan la transacción, excluyendo a cualquier tercero o intermediario. (2) La economía colaborativa también tiene como objetivo reducir el desperdicio y la sobreproducción de artículos. Fomenta ciudades limpias y sostenibles al permitir que los consumidores obtengan acceso a los mismos productos a partir de un gran conjunto de recursos disponibles a través de redes en línea, en lugar de productos nuevos que consumen mucho (Gansky, 2010). Además (3), la agregación eficiente de innovaciones tecnológicas en las formas colaborativas de producción y consumo permite ampliar el alcance de todo el esquema de local a global. Internet contribuye al desempeño de la colaboración a gran escala (Sundararajan, 2016). Finalmente (4), la economía colaborativa abarca tanto las prácticas cooperativas como la construcción comunitaria (Felson y Spaeth, 1978; Lessig, 2008; Matofska, 2016). Concretamente, este modelo de consumo afirma que al realizar un consumo colaborativo aumentan los niveles de felicidad y satisfacción personal.

Sin embargo, se ha observado que, dada la novedad de la economía colaborativa, todavía faltan investigaciones empíricas que apunten a comprender el comportamiento comercial, cultural, social y tecnológico de este paradigma perturbador. Acercándose a un alcance más amplio, desde mediados de los años 60, se han publicado múltiples estudios que intentan comprender los factores clave por los cuales los individuos se involucran en comunidades colaboradoras, así como las posibles causas que propician actos de egoísmo y egoísmo. Por lo tanto, para construir una narrativa más explicativa en la que se conecten modelos de cooperación fuera de línea y en línea, este estudio se desarrolla de la siguiente manera. Primero, explore las teorías más relevantes sobre la cooperación humana, teniendo también en cuenta las investigaciones basadas en comportamientos racionales (1965-2010). Y segundo, analizar y argumentar las diversas definiciones de economía colaborativa propuestas por varios expertos en este campo (20042016). Este trabajo concluye con su conclusión teórica en la que las principales preguntas de investigación son cuidadosamente argumentadas. Estos son: ¿es posible explicar la naturaleza colaborativa de la economía colaborativa a partir de teorías notables (fuera de línea) sobre la cooperación humana? ¿Qué tipo de similitudes y diferencias se encuentran al comparar teorías bien establecidas sobre la cooperación humana y la economía innovadora de intercambio? 


\section{METODOLOGÍA}

Dada la naturaleza teórica de esta investigación, se utilizan referencias bibliográficas notables como recurso primario de investigación. Tomando el año 1965 como punto de partida, tiene como objetivo recopilar teorías altamente relevantes sobre la cooperación humana, así como sobre acciones egoístas y racionales; a su vez, sus principios más sustanciales serán evaluados y expuestos. Además, para contextualizar la economía colaborativa como un sistema colaborativo y digital, este documento procederá a recopilar, explorar y ordenar cronológicamente nueve definiciones de este sistema socioeconómico disruptivo establecido por Benkler, (2004), Tapscott y Williams (2006), Algar (2007), Lessig (2008), Botsman y Rogers (2010), Bauwens, (2012), Rifkin (2014), Stephany (2015) y Sundararajan (2016). Esta información se organizará por medio de una tabla en la que se presentarán la cita original del autor, el año de publicación y los principales puntos de vista. Al combinar todas las partes esenciales, la investigación logrará una narración más explicativa en la que se expondrán los hallazgos de la investigación.

\section{EXPLORANDO TEORÍAS SOBRE LA COOPERACIÓN HUMANA (FUERA DE LÍNEA).}

\subsection{Teorías sobre la acción colectiva y la tentación de viajar gratis.}

Las teorías más relevantes sobre la acción colectiva se escribieron entre los años 60 y 70, un período en el cual las opiniones diferían mucho en diferentes ubicaciones geográficas. Los estudios estadounidenses se basaron en micro niveles de investigación que otorgaron mayor importancia a la relación entre individuos. Como resultado, los académicos desarrollaron la teoría de movilización de recursos (RMT). Esta teoría define los movimientos sociales como intercambios racionales, oponiéndose a la teoría tradicional del comportamiento colectivo, que es conocida por su irracionalidad. Los movimientos sociales construidos bajo RMT siguen los objetivos políticos al recolectar recursos financieros y atraer la atención de los medios. Charles Tilly y Doug McAdam fueron escritores importantes de esta teoría política (Kendall, 2006).

En ese momento en Europa, los académicos preferían estudiar los movimientos sociales usando técnicas macro y priorizando a la sociedad en general. Los críticos desarrollaron la teoría de los nuevos movimientos sociales (NSM), que se centra en el análisis de los derechos sociales. Esta teoría estaba ligada a cuestiones contemporáneas, por ejemplo, las economías postindustriales o el capitalismo tardío (Diani y Eyerman, 1992; Pichardo, 1997).

En particular, Olson (1965), Hardin (1968) y Ostrom (1990) han hecho una gran contribución teórica a la comprensión de los bienes comunes y su gestión por parte de las comunidades. Muchos de sus estudios exploran cómo las personas interactúan dentro de grupos y redes para obtener el máximo beneficio de los recursos naturales, bienes o servicios. Por lo tanto, en un esfuerzo por vincular estas teorías a la colaboración de la economía colaborativa, este trabajo procede a examinar cuidadosamente y resumir los principales hallazgos de, no solo los enfoques desarrollados por estos tres estudiosos, sino también otras investigaciones notables 
sobre la cooperación humana y el egoísmo emitidos entre el siglo 20 y principios del siglo 21.

El documento "The Tragedy of the Commons", publicado en 1968 por Garrett Hardin, generó críticas dentro de la comunidad científica. El enfoque de Hardin (1968) describe una situación en la que un número de individuos, motivados únicamente por sus propios intereses y comportamiento racional, conducen a la destrucción de sus bienes comunes. En su opinión, esta situación ocurrirá incluso cuando los miembros pretendan proteger sus recursos compartidos. El estudioso llama la atención sobre un caso en el que un pasto de acceso abierto es explotado por agentes racionales. En este caso, el individuo egoísta tenderá a agregar más animales para pastar la hierba, a fin de aumentar su propio beneficio. Este individuo obtendrá un ingreso más alto mientras que el costo total de mantenimiento del pasto será compartido por todos los miembros. Finalmente, el pasto será destruido por la gran cantidad de animales. Finalmente, en opinión de Hardin, el campo común llegará a su tragedia. El investigador atribuyó este problema como una consecuencia directa de las economías racionales y advirtió sobre lo negativo que puede ser para los recursos naturales:

Ahí está la tragedia. Cada hombre está encerrado en un sistema que lo obliga a aumentar su manada sin límites, en un mundo limitado. La ruina es el destino hacia el cual todos los hombres se apresuran, cada uno persiguiendo su propio interés en una sociedad que cree en la libertad de los comunes. (Hardin, 1968)

Tres años antes de la teoría de Hardin, Olson (1965) publicó sus hallazgos en su libro titulado "La lógica de la acción colectiva". Este libro enfatiza el comportamiento lógico de las personas que participan en grupos mediante incentivos como un medio de motivación. Para él, un grupo de individuos que actúen colectivamente estaría tentado a viajar gratis solo cuando el grupo esté utilizando bienes públicos. Sin embargo, si los beneficios obtenidos solo los disfrutan los miembros del grupo, no actuarán individualmente. Además, en estos casos, es menos probable que los grupos más grandes participen en acciones colectivas debido a que enfrentarán costos más altos y las personas obtendrán ingresos reducidos, Olson (1965) declaró:

... si los miembros de algún grupo tienen un interés u objeto en común, y si todos estuvieran mejor si se lograra ese objetivo, se ha pensado lógicamente que las personas de ese grupo, si fueran racionales y autónomos, interesado, actúe para lograr ese objetivo... (Olson, 1965)

Además de enfoque de Olson, los estudiosos Kaplan y Gurven (2001) identifican tres temas influyentes que mejorarían el parasitismo en grupos de colaboración. En primer lugar, como Olson (1965) creía, el tamaño del grupo es proporcional a la posibilidad de montar a caballo libre, ya sea debido a un alto número de usuarios aumentan automáticamente el número posible de pilotos libres o porque en grandes grupos la posibilidad de ser acusado de no cooperar con otros disminuye. En segundo lugar, si las personas tienen acceso a datos de buena calidad sobre transacciones, ganancias, pérdidas e información personal de otros miembros, esta podría ser una gran herramienta para practicar el free riding entre otros. Y, por último, los grupos en los que hay un alto nivel de no parientes se vuelven sujetos al egoísmo (véase también Cosmides y Tooby, 1992). 
En Hardin (1968) y Olson (1965), la acción colectiva y la construcción de la comunidad surgen de un dilema social crucial: un individuo de un grupo solo obtendrá beneficios cuando el resto de los miembros colabore también, sin embargo, si otros miembros tienden a liberar montar este individuo podría ver sus inversiones y beneficios futuros en peligro. Asumiendo que la colaboración de otros miembros no está garantizada, la persona inicial podría estar involucrada en un dilema personal, una confrontación entre su propio interés racional y los mejores resultados para todo el grupo.

Deconstruyendo los enfoques de Hardin y Olson, Ostrom (1990) desarrolló un estudio complejo llamado "Governing the Commons" (Ganador del Premio Nobel 2009) cuestionando la eficacia de tales teorías. Para Ostrom (1990), los grupos autónomos y autogestionados pueden alcanzar grandes niveles de cooperación mientras previenen la tragedia de los comunes. Además, afirma que ni el estado ni el mercado son lo suficientemente favorables para conectar individuos y recursos comunes. Ella agrega que "se percibe a los individuos como atrapados en una situación estática, incapaces de cambiar las reglas que afectan sus incentivos". Ostrom (1990) basa su investigación en estudios de casos profundos donde, en su opinión, primero, a lo largo de la historia muchos exitosos las instancias de manejo de recursos naturales ocurrieron sin ninguna intermediación por parte del estado o de organizaciones privadas, y segundo, los derechos de propiedad no garantizan el mantenimiento de los bienes comunes. De hecho, Ostrom (1990) niega que las políticas gubernamentales ayuden a los miembros de un grupo a organizarse. Ostrom (1990) propone un modelo alternativo basado en el aprendizaje en el cual los individuos monitorean y adoptan estrategias del resto de los actores, recopilando la información requerida para alcanzar el objetivo común. Si este proceso continúa a lo largo del tiempo y los participantes han desarrollado "normas de reciprocidad" compartidas, es más probable que adquieran estrategias comunes de autogestión.

Desde un punto de vista diferente, Kaplan y Gurven (2001) sostienen que los individuos racionales y egoístas solo se comprometerán con el altruismo recíproco cuando las recompensas y los castigos se repitan y refuercen continuamente. Los actores que intentan aprovechar los recursos comunes mediante el comercio o la negociación deben ser reprendidos (Kaplan y Gurven, 2001). Este argumento está respaldado por hallazgos previos de Boyd y Richerson (2005) que afirman que la cooperación humana está dominada principalmente por la penalización. En su opinión, las comunidades colaborativas más grandes solo pueden resistir cuando se ejecutan altos niveles de castigo, por lo tanto, aquellos que desobedecen o los colaboradores que no castigan a los desertores deben ser castigados. Muchos experimentos sociales se han desarrollado sobre la base de la recompensa y el castigo entre los jugadores. Este documento presta especial atención al juego del dilema de los presos.

Axelrod y Hamilton (1981) han centrado su investigación en la comprensión de cómo la cooperación y el egoísmo se desarrollan entre individuos. La base teórica y experimental para su estudio se basa principalmente en la teoría de la acción racional y los principios del juego del dilema del prisionero, originalmente diseñado por Merrill M. Flood y Melvin Dresher (1950). Este dilema propone una situación en la que dos personas son acusadas de haber cometido un crimen, pero ninguna de ellas tiene pruebas suficientes para demostrar su inocencia. Ambos son arrestados y 
encarcelados en celdas separadas. Deben tomar una decisión que genere ganancias individuales o beneficios colectivos. La selección en este juego se toma individualmente $y$ en privado, los presos pueden: cooperar, lo que significa permanecer en silencio, o desertar y acusar al otro (Poeete, Janssen \& Ostrom, 2010). El juego del dilema del prisionero establece las siguientes reglas:

Tabla 1

Juego de dilema del prisionero.

\begin{tabular}{ccc}
\cline { 2 - 3 } & $\begin{array}{c}\text { Prisionero A } \\
\text { Coopera }\end{array}$ & $\begin{array}{c}\text { Prisionero A } \\
\text { Defectos }\end{array}$ \\
\hline $\begin{array}{c}\text { Prisionero } \\
\text { B }\end{array}$ & $\begin{array}{c}\text { Ambos son condenados por 1 } 1 \\
\text { año. }\end{array}$ & $\begin{array}{c}\text { El prisionero A queda en libertad } \\
\text { y el prisionero B es condenado } \\
\text { poopera } 5 \text { años. }\end{array}$ \\
\hline $\begin{array}{c}\text { Prisionero } \\
\text { B }\end{array}$ & $\begin{array}{c}\text { El prisionero B sale libre y el } \\
\text { prisionero A es condenado } \\
\text { pefectos }\end{array}$ & $\begin{array}{c}\text { Ambos son condenados por 3 } 3 \\
\text { años. }\end{array}$ \\
\hline
\end{tabular}

El mejor caso para el grupo sería aquel en el que ambos socios cooperan. Sin embargo, este juego razona que ambos son actores racionales egoístas $\mathrm{y}$, por lo tanto, están tratando de obtener la oración mínima posible: el Prisionero A pensaría que si el Prisionero B coopera, entonces es mejor desertar y quedar libre, mientras que si el Prisionero B falla, la mejor opción es desertar también, dado que tres años es mejor que cinco. Por lo tanto, este dilema sostiene que una persona racional tenderá a desertar, incluso suponiendo que su pareja pueda sufrir la peor sentencia.

El juego del dilema del prisionero, así como muchos otros experimentos sociales, demuestra que no siempre hay un consenso sobre la cooperación. Tales enfoques indican que las elecciones humanas se basan principalmente en intereses personales y, por lo tanto, una comunidad estaría conformada por las decisiones racionales individuales de cada miembro. Como tal, la metáfora de "la mano invisible" escrita por Smith (1759) describe cómo los individuos, como tomadores de decisiones, pueden generar colectivamente un sistema económico efectivo. En una situación gobernada por la mano invisible; los vendedores, compradores, productores e importadores tienen su propio derecho a equilibrar las ganancias y pérdidas estableciendo los precios. Cada jugador ejecuta elecciones individuales para maximizar su beneficio personal, liberando así el mercado (Smith, 1759).

Usando teorías evolutivas y de selección, Kaplan y Gurven (2001) identifican ocho hechos clave que, en algún momento, las personas experimentan cuando cooperan dentro de una comunidad; (1) desarrollan una percepción especial para detectar ganancias potenciales; (2) buscarán la forma de obtener un beneficio de estos logros; (3) tenderán a andar libremente; y, a su vez (4) evitarán ser víctimas de la libertad de andar; (5) Desarrollarán la perspicacia requerida para encontrar oportunidades para el viaje libre y (6) desarrollarán la sensibilidad para detectar y analizar los costos personales y los resultados grupales actuando colectivamente; (7) los actores racionales realizarán habilidades para negociar interna y común normas para maximizar ganancias personales y; (8) apuntarán a ejecutar obedientemente las 
normas mientras animan a otros a actuar de manera similar para que se respete el castigo.

\subsection{Introducción al consumo colaborativo (fuera de línea) y algunos factores relacionados.}

Para concluir esta sección específica y tratar de unir las teorías anteriores y la economía compartida de transmisión digital, este estudio procede a observar la primera definición del concepto de consumo colaborativo (un término que también se usa hoy en día para referirse a la economía colaborativa) encontrado hasta este hora. En 1978, Marcus Felson y Joe L. Spaeth acuñaron el término consumo colaborativo en su documento "Estructura de la comunidad y consumo colaborativo" sobre el uso compartido del automóvil. Prestaron especial atención a las interacciones humanas y descubrieron que las personas prefieren hacer las cosas en grupos porque generalmente se logra un objetivo común de manera rápida y fácil por consenso mutuo. Más concretamente, Felson y Spaeth (1978) proponen la siguiente definición:

Consumo colaborativo, a saber, aquellos eventos en los que una o más personas consumen bienes o servicios económicos en el proceso de participar en actividades conjuntas con uno o más de otros. Por ejemplo, beber cerveza con amigos, comer con familiares, conducir para visitar a alguien o usar una lavadora para lavar la ropa de la familia son actos de consumo colaborativo (p. 614).

El consumo colaborativo, en su opinión, se centra principalmente en aspectos del estilo de vida, la construcción de la comunidad y el comportamiento del consumidor. El tiempo también representa un factor crucial dado que las actividades de rutina cooperativas están vinculadas a la coordinación temporal. Además, la estructura espacio-temporal de una comunidad afecta directamente el desarrollo de actividades de consumo colaborativo, como comer, conducir o aprender. Por ejemplo, una comunidad que realiza las mismas actividades de rutina en intervalos de tiempo similares tenderá a involucrarse mejor con el consumo colaborativo. Por ejemplo, las horas de trabajo o los horarios escolares influirán en actividades como almorzar con compañeros de trabajo, ir al parque con otros padres y sus hijos, hacer autostop, etc.

Esta definición temprana, que más tarde sería mejor conocida como la economía colaborativa, descarta cualquier tipo de tecnología como herramienta para fomentar la cooperación o la construcción de la comunidad. Sin embargo, sugiere que las interacciones sociales, los sentimientos, las experiencias personales y los intereses similares fomentan enormemente la formación de grupos impulsados por la colaboración.

De manera similar, Diani y Eyerman (1992) definen las redes de movimientos sociales contemporáneos como las relaciones e intercambios entre individuos y organizaciones que comparten las mismas creencias y que están constituidas por una cierta identidad colectiva. Atribuyen "el sentido de pertenencia" como una característica fuerte de las comunidades sociales junto con la "unidad". En su opinión, la seguridad de la supervivencia de la comunidad requiere interacciones 
constantes entre los participantes; intercambios, debates, comentarios y negociaciones. A diferencia de Olson (1965), quien argumentó que la heterogeneidad obstaculiza la cooperación dentro de un grupo, Diani y Eyerman (1992) advierten a los investigadores sociales sobre la dificultad de encontrar las fronteras de las comunidades dado que no son "entidades empíricas" sino actores heterogéneos ( Ver también Ostrom, Jansen y Poeete , 2010).

Muchos expertos en economía compartida consideran que la confianza y la reputación (por ejemplo, Botsman y Rogers, 2010, Owyang, 2013, Gansky, 2010) son vitales para el desarrollo óptimo del consumo colaborativo. En consecuencia, Arrow (1974) destaca la importancia de la confiabilidad y la reputación positiva como factores clave en la construcción de la comunidad; él afirma que "[confiar] es extremadamente eficiente, ahorra muchos problemas para tener un buen grado de confianza en la palabra de otras personas". Del mismo modo, Ostrom (2010) agrega que para lograr un nivel óptimo de participación, los miembros tienen Desarrollar confianza a partir de un proceso de aprendizaje recíproco, por lo tanto, cuando los actores de una determinada comunidad repiten acciones colectivas a lo largo del tiempo, es más probable que otros aprendan y adopten estas acciones. Si se continúa este proceso, ella afirma, "los niveles de confianza y reciprocidad se reforzarán mutuamente". Además, Ostrom (2010) identifica ciertas variables para aumentar la confianza en las redes y las comunidades, a saber: (a) la reputación de los miembros debe ser conocida por todo el grupo, cuando los miembros publican y comparten la información, los niveles de cooperación aumentan; (b) los debates personales y cara a cara conducen a la confiabilidad y; (c) la comunidad debe garantizar a los miembros el derecho de ingresar y salir en cualquier momento.

\section{EXPLORACIÓN DE LA COLABORACIÓN HUMANA (EN LÍNEA): LA ECONOMÍA COLABORATIVA}

En este punto, este documento cambia su alcance para explorar únicamente enfoques basados en la colaboración mediante tecnologías digitales, fundamentalmente a través de plataformas digitales. Es de gran importancia notar que aunque las prácticas colaborativas y de intercambio datan de la antigüedad, la economía colaborativa es solo comprensible dentro de un contexto digital (Sundararajan, 2016, Howard, 2015; Stephany, 2015).

La Tabla 2 recopila nueve de las definiciones más influyentes de la economía colaborativa desde 2004 hasta 2016. Aunque los autores difieren al nombrar este fenómeno disruptivo, su visión sobre lo que representa la economía colaborativa es ampliamente compartida por todos ellos. La Tabla 3 pretende exponer cronológicamente no solo las citas directas de once renombrados expertos en economía colaborativa (Benkler, 2004; Tapscott y Williams, 2006; Algar, 2007; Lessig, 2008; Botsman y Rogers, 2010; Bauwens, 2012; Rifkin, 2014; Stephany , 2015; Sundararajan, 2016), pero también las ideas más destacadas que se encuentran en toda su literatura. 
Martos Carrión, E. De la colaboración fuera de línea a la línea: enfoque teórico sobre los estudios de cooperación humana y la economía colaborativa

\section{Tabla 2}

Análisis de las interpretaciones de la economía colaborativa obtenidas de la literatura relevante (2004-2016).

\begin{tabular}{|c|c|}
\hline $\begin{array}{l}\text { Año / Autor } \\
\text { / Término } \\
\text { propio }\end{array}$ & Cita del autor y principales ideas. \\
\hline $\begin{array}{l}2004 \\
\text { Yochai Benkler } \\
\text { Productos } \\
\text { compartibles y } \\
\text { producción de } \\
\text { pares basada } \\
\text { en los } \\
\text { comunes }\end{array}$ & $\begin{array}{l}\text { "(Prácticas / bienes compartibles) representan instancias en las que } \\
\text { el intercambio social es totalmente impersonal o entre personas } \\
\text { poco afiliadas que participan en prácticas sociales que implican } \\
\text { contribuciones de la capacidad de sus bienes privados en patrones } \\
\text { que se combinan para formar sistemas eficaces y de gran escala } \\
\text { para aprovisionamiento bienes, servicios y recursos "(p.275-276) } \\
\text { - La redistribución de los recursos se basa en relaciones y ética } \\
\text { distribuidas más que en propósitos económicos } \\
\text { - Las tecnologías permiten la posibilidad de construir redes } \\
\text { descentralizadas de base social opuestas a los mercados } \\
\text { jerárquicos. }\end{array}$ \\
\hline $\begin{array}{l}2006 \\
\text { Don Tapscott y } \\
\text { Anthony D. } \\
\text { Williams } \\
\text { Wikinomics y } \\
\text { economía de } \\
\text { colaboración }\end{array}$ & $\begin{array}{l}\text { "Creación y comunicación punto a punto, esta fuerza totalmente } \\
\text { descentralizada y amorfa se autoorganiza cada vez más para } \\
\text { proporcionar sus propias noticias, entretenimiento y servicios. A } \\
\text { medida que estos efectos se difunden a través de la economía y se } \\
\text { cruzan con profundos cambios estructurales como la globalización, } \\
\text { asistiremos al surgimiento de un tipo de economía completamente } \\
\text { nuevo donde las empresas coexisten con millones de productores } \\
\text { autónomos que se conectan y crean valor conjuntamente en redes } \\
\text { débilmente acopladas. Llamamos a esto la economía colaborativa } \\
\text { "(p.32). } \\
\text { - Las nuevas tecnologías y los sistemas operativos innovadores } \\
\text { desempeñan un papel esencial en el desarrollo de la economía } \\
\text { colaborativa y la wikinomics. } \\
\text { - Internet sirve como una infraestructura de bajo costo para la } \\
\text { colaboración y la producción entre pares. }\end{array}$ \\
\hline $\begin{array}{l}\text { Consumo } \\
\text { colaborativo }\end{array}$ & $\begin{array}{l}\text { "¿Qué sucede cuando la información sobre precios se vuelve } \\
\text { accesible y los consumidores comienzan a compartir el } \\
\text { conocimiento? Bienvenido al mundo del consumo colaborativo (...) } \\
\text { La tecnología está democratizando el proceso de compra y, en } \\
\text { última instancia, los consumidores decidirán hasta qué punto los } \\
\text { proveedores de ocio pueden superar los límites de estas formas } \\
\text { ingeniosas de optimizar los precios. El individuo se ha transformado } \\
\text { en una multitud y la multitud es sabia "(p.16-17). } \\
\text { - El consumo colaborativo es un fenómeno global que aprovecha la } \\
\text { conectividad. } \\
\text { - Los consumidores colaboran a través de la web para intercambiar } \\
\text { bienes y servicios. La colaboración con la multitud aporta mucho } \\
\text { más que actuar individualmente. }\end{array}$ \\
\hline $\begin{array}{l}2008 \\
\text { Lawrence } \\
\text { Lessig } \\
\text { Economías } \\
\text { compartidas y }\end{array}$ & $\begin{array}{l}\text { "Existe no solo la economía comercial, que mide el acceso en la } \\
\text { simple métrica del precio, sino también una economía de } \\
\text { intercambio, donde el acceso a la cultura no está regulado por el } \\
\text { precio, sino por un complejo conjunto de relaciones sociales. Estas } \\
\text { relaciones sociales no son simples. (...) todos los que lean este libro } \\
\text { tienen una rica vida de relaciones regidas en una economía de }\end{array}$ \\
\hline
\end{tabular}




\begin{tabular}{|c|c|}
\hline $\begin{array}{l}\text { economías de } \\
\text { uso } \\
\text { compartido de } \\
\text { Internet }\end{array}$ & $\begin{array}{l}\text { intercambio, libres de la simplicidad de los precios y los mercados". } \\
\text { (p.145) } \\
\text { - Compartir las economías mejora las conexiones personales y las } \\
\text { comunidades cooperativas. } \\
\text { - Compartir y las economías comerciales se complementan entre sí. } \\
\text { - Al incorporar Internet, las economías compartidas amplían su } \\
\text { alcance y se vuelven globales y abarrotadas. }\end{array}$ \\
\hline $\begin{array}{l}2010 \\
\text { Rachel } \\
\text { Botsman y Roo } \\
\text { Rogers } \\
\text { Consumo } \\
\text { colaborativo }\end{array}$ & $\begin{array}{l}\text { "El consumo colaborativo permite a las personas darse cuenta de } \\
\text { los enormes beneficios del acceso a los productos y el servicio } \\
\text { sobre la propiedad, y al mismo tiempo ahorrar dinero, espacio y } \\
\text { tiempo, hacer nuevos amigos y volverse ciudadanos activos" (p. } \\
\text { - Es un modelo económico en el que los activos infrautilizados se } \\
\text { intercambian, alquilan o intercambian. Los sistemas de reputación } \\
\text { son cruciales. } \\
\text { - El consumo colaborativo depende de redes en línea y nuevos } \\
\text { dispositivos tecnológicos. } \\
\text { - Siglo XX vs. XXI: el consumo hiper vs consumo colaborativo, el } \\
\text { crédito frente a la reputación, la publicidad frente a la comunidad y } \\
\text { la propiedad contra el acceso compartido. }\end{array}$ \\
\hline
\end{tabular}

2012

Michel

Bauwens

Economía colaborativa $y$ producción de pares
(1) Inmaterial: "En la economía colaborativa inmaterial, lo que se mutualiza es el conocimiento, el software y el diseño, a través de recursos compartidos comunes de innovación generalmente gobernados por licencias legales específicas" (p.51)

(2) Material: "El ámbito de rápido crecimiento del consumo colaborativo utiliza plataformas de servicios de productos, a menudo bajo propiedad corporativa, que permiten a los usuarios y consumidores el acceso colectivo a bienes físicos". (P.53)

(3) Mixto: "Plataformas en las que los diseñadores de productos tanto inmateriales como materiales pueden ofrecer su trabajo a la venta, pero donde tiene lugar alguna forma de agregación o filtrado colectivo" (p.52)

- La integración de las redes sociales y las innovaciones digitales funciona como una herramienta notable para el desarrollo exponencial de la economía colaborativa
2014

Jeremy Rifkin

Collaborative Commons
"Conectar a todos y todo en una red global impulsada por una productividad extrema nos mueve cada vez más rápido hacia una era de bienes y servicios casi gratuitos $y$, sin el golpe del capitalismo en el próximo medio siglo y el surgimiento de Collaborative Commons como el modelo dominante para organizar la vida económica (...) donde miles de millones de personas se involucran en los aspectos profundamente sociales de la vida "(p.16).

- La alta conectividad de los sistemas operativos innovadores como Internet de las cosas representa el declive de las economías capitalistas y el aumento de los bienes comunes de colaboración.

Las nuevas tecnologías incrustan la producción y el consumo global de pares en una "cultura de intercambio".

2015

"La economía colaborativa es el valor de tomar activos subutilizados

Alex Stephany

El negocio de compartir y hacerlos accesibles en línea a una comunidad, lo que lleva a una menor necesidad de propiedad de esos activos" (p.9).

- Las plataformas de economía colaborativa suelen ser redes basadas en el mercado que generan un valor económico recíproco. 


\begin{tabular}{ll}
\hline & $\begin{array}{l}\text { Los sistemas de reputación juegan un papel importante. } \\
\text { La economía colaborativa se ha convertido en un marco en línea. } \\
\text { Internet es vital para su surgimiento. }\end{array}$ \\
\hline 2016 & "Yo llamo a la economía colaborativa o al capitalismo basado en la \\
Arun & multitud, términos que uso más precisamente para describir un \\
Sundararajan & $\begin{array}{l}\text { ecosistema impulsado por las siguientes cinco características: } \\
\text { capital basado en el mercado, de gran impacto, redes basadas en }\end{array}$ \\
$\begin{array}{l}\text { Capitalismo } \\
\text { basado en la } \\
\text { muchedumbre }\end{array}$ & $\begin{array}{l}\text { multitud, línea difusa entre lo personal y lo tanto profesional como } \\
\text { desdibujando la línea entre el trabajo totalmente empleado y el } \\
\text { ocasional "(p.26-27). }\end{array}$ \\
& $\begin{array}{l}\text { Este nuevo fenómeno genera mercados innovadores en línea } \\
\text { donde los activos son potencialmente intercambiados } \\
-\quad \text { La economía colaborativa o el capitalismo basado en la multitud } \\
\text { amplía el uso de productos al crear nuevas oportunidades para } \\
\text { compartir. }\end{array}$ \\
\hline
\end{tabular}

La Tabla 2 destaca que, fundamentalmente, todos los significados de la economía colaborativa consideran que las innovaciones digitales son primordiales cuando se desarrolla este paradigma socio-económico. Las definiciones señalan que la economía colaborativa, como se observó anteriormente, no habría existido si no fuera por la transformación de la página web inicial en plataformas comerciales. Además, la mayoría de los expertos en economía colaborativa también están de acuerdo en el hecho de que las redes descentralizadas moldean la estructura interna de esta economía, lo que permite que las personas se conviertan en responsables de la toma de decisiones. Además, el pase de propiedad debe ser reemplazado por el acceso, es decir, la economía colaborativa proporciona a sus usuarios una gran cantidad de recursos que, normalmente por una tarifa, los pares pueden acceder por un tiempo limitado, anulando a su vez la necesidad de compra. Y, por último, las definiciones otorgan que el campo de estudio esencialmente opera a escala global.

Después de haber explorado diversas concepciones de la llamada economía colaborativa, se ha abordado una comprensión más precisa sobre, no solo cómo se configura, sino también sus objetivos y características principales. En consecuencia, este documento procede a comparar y analizar esta comprensión con respecto al conjunto de enfoques sobre cooperación humana estudiados en la sección 3.

\section{DISCUSIÓN Y CONCLUSIÓN}

A lo largo de décadas anteriores, las prácticas de intercambio y los comportamientos colaborativos han sido cuestiones imperativas para muchos investigadores que intentan comprender la naturaleza de las interacciones entre los miembros de la misma red. Al desarrollar este estudio, en el que se han explorado muchas teorías importantes sobre acciones colectivas / egoístas y definiciones de economía colaborativa, este documento ha encontrado algunos hallazgos relevantes con respecto a las preguntas de investigación planteadas al comienzo de este documento. Por lo tanto, y en un intento de comprender qué tipo de conexiones pueden ser un puente entre los enfoques populares sobre la cooperación (fuera de línea) y la economía colaborativa, este documento procede a enumerar sus observaciones más esenciales. 
1. Aunque la mayoría de los académicos mencionados en este trabajo acuerdan cómo están estructuradas las comunidades (es decir, como un conjunto de individuos, intercambios e interacciones formadas por creencias, valores u objetivos comunes), varían ampliamente en su razonamiento sobre por qué y cómo los actores cooperan entre ellos. Uno de los hechos que parece más discutido acerca de si fomenta la cooperación dentro de una comunidad es el tamaño del grupo. Kaplan y Gurven (2001), así como Olson (1965), afirman con fuerza que los grupos grandes tienen menos probabilidades de generar colaboración que los grupos pequeños, de hecho, los miembros de comunidades importantes tenderán a viajar libremente. Sin embargo, se ha encontrado con certeza que la economía colaborativa funciona a escala global (Sundararajan, 2016; Rifkin, 2014; Lessig, 2008), lo que sugeriría que las comunidades grandes también pueden desarrollar altos niveles de colaboración. De hecho, varias plataformas consideradas como parte de la economía colaborativa, como Bitcoin y Wikipedia, solo son potencialmente efectivas cuando sus comunidades logran una cantidad sustancial de usuarios. Si la comunidad no es lo suficientemente grande, podrían desaparecer porque no pueden brindar su servicio con éxito. Este tipo de plataformas de intercambio descentralizadas se basa en la abundancia, todos los pares contribuyen a generar dicha abundancia ya sea cargando contenido, ofreciendo sus servicios o realizando cualquier otra tarea comunitaria. Por lo tanto, cuanto más grande sea la red, mejor.

2. La heterogeneidad también es una característica sujeta a debate. Olson (1965) y Kaplan y Gurven (2001) consideran que los grupos formados por actores de diferentes culturas, edades, razas y creencias realizan menos niveles de colaboración en comparación con las comunidades homogéneas. Por otro lado, Diani y Eyerman (1992) sugieren que los miembros de la comunidad son fundamentalmente colaborativos porque un cierto sentido de identidad los motiva a hacerlo. Al abordar las definiciones de economía colaborativa, es importante observar que las redes de colaboración, debido a sus grandes tamaños y su naturaleza virtual, están principalmente formadas por actores heterogéneos (Sundararajan, 2016). Por lo tanto, podría existir, como plantean Diani y Eyerman (1992), un objetivo compartido sobre todos los usuarios particulares que los alienta a involucrarse en la colaboración. Por ejemplo, los colaboradores de Linux cooperan voluntariamente intentando desarrollar un sistema operativo de código abierto eficiente y adecuado a sus necesidades (Benkler, 2004). Del mismo modo, la red de economía colaborativa Waze (www.waze.com), que permite a los usuarios actualizar en tiempo real, mapas de carreteras, saltos de tráfico, accidentes automovilísticos, etc., se basa completamente en la información que los pares proporcionan libremente. Los miembros de esta red altamente heterogénea y global colaboran por el bien común de estar al día cuando se refieren a problemas de tráfico.

3. Por lo tanto, como se indicó anteriormente, los usuarios de la economía colaborativa esperan cualquier tipo de compensación cuando colaboran dentro de dichas redes. La mayoría de los académicos estudiados en la primera parte de 
este documento coinciden en que, de una forma u otra, los colaboradores se sienten impulsados por las recompensas (Olson, 1965, Kaplan y Gurven, 2001, Boyd y Richerson, 2005). Los enfoques de estos autores, que fueron construidos para explicar los paradigmas fuera de línea, también son aplicables al paradigma de la economía colaborativa. Todos los expertos en economía colaborativa observan que este modelo digital de intercambio no es altruista y, por lo tanto, los usuarios esperarán ser compensados en algún momento. Más concretamente, a diferencia de Lessig (2008) y Benkler (2004) que creen que las economías compartidas no son comerciales, sin embargo, impulsados por beneficios y propósitos sociales y emocionales, el resto de los autores de la economía colaborativa consideran que este fenómeno disruptivo se basa fundamentalmente en el deseo de obtener ganancias económicas.

4. Después de haber explorado enfoques significativos centrados en la cooperación humana, se ha descubierto que algunos estudiosos (por ejemplo, Boyd y Richerson, 2005; Kaplan y Gurven, 2001; Axelrod y Hamilton, 1981 ) consideran que las recompensas y el castigo prácticamente funcionan de manera similar cuando viene a generar cooperación Experimentos como el juego del dilema del prisionero demuestran que, en la mayoría de las ocasiones, el castigo y las recompensas influyen en gran medida en las respuestas de las personas cuando se trata de cooperar o desertar. Cabe señalar que, aunque este trabajo considera que este experimento específico no es directamente aplicable a la comprensión de la economía colaborativa en su conjunto, debido a que los usuarios de la economía compartida cooperan a priori libremente y ningún control externo los obliga a participar en consumos colaborativos, se ha encontrado una gran analogía entre castigos / recompensas y sistemas de clasificación en línea. Boyd y Richerson (2005) afirman que los miembros de una comunidad están a cargo de informar actividades negativas realizadas por otros miembros. Al hacer eso, todos los pares son conscientes de que serán castigados si cometen cualquier acto inaceptable. Aplicando esta declaración al paradigma de la economía colaborativa, los sistemas de reputación, que parecen crear un modelo de gobierno más justo y confiable (Botsman y Rogers, 2010; Stephany, 2015; Matofska, 2016), podrían funcionar como la versión en línea de Boyd y Richerson supuesto (2005). Los sistemas de reputación permiten a los usuarios de la economía compartida calificar y comentar los servicios ofrecidos por otros usuarios, ya sea que hayan sido satisfactorios o no. En ese sentido, los sistemas de reputación operan como un canal para recompensar o castigar a los miembros de la comunidad. Aquellos que hayan realizado alguna práctica negativa serán castigados con malas críticas y disminuirán sus posibilidades de brindar sus servicios nuevamente. Estos sistemas de calificación son públicos y accesibles para cualquier miembro de la comunidad, por lo tanto, lograr una puntuación positiva cuando intercambiar, alquilar, intercambiar o intercambiar cualquier bien o servicio es primordial para los usuarios de la economía compartida que dependen en gran medida de su reputación en línea (ver también Slee, 2015)

5. Los sistemas de reputación están destinados a desarrollar la confianza entre los miembros desconocidos de las redes de economía colaborativa (Howard, 2015; 
Gansky, 2010; Owyang, 2013). Como ya se mencionó, estas redes funcionan principalmente a escala global, lo que implica que los colaboradores son prácticamente desconocidos. En estudios desarrollados por Kaplan y Gurven (2001) y Ostrom (2010), la cooperación requiere confianza y esto se logrará mejor con los familiares y a través de las relaciones cara a cara. Tales argumentos serían insostenibles al explicar cómo se maneja el concepto de confianza en el esquema de economía digital compartida. Se ha demostrado que las plataformas globales de economía colaborativa generan altos niveles de confianza, lo que a su vez implica que los usuarios practiquen activamente la colaboración (Botsman y Rogers, 2010). Por lo tanto, es importante notar que, la confianza parece haber sido reinterpretada en una forma digital que amplía enormemente su alcance de lo personal a lo impersonal y de lo local a lo global. Además de eso, ha habido controversia cuando se trata de explicar si la información pública y transparente fomenta la cooperación o no. Kaplan y Gurven (2001) argumentan que el acceso común a la información personal de otros, ingresos, pérdidas, transacciones, etc. podría funcionar como una poderosa herramienta para que los individuos puedan usar a otros. En otras palabras, un actor puede usar esta información contra otros miembros en un intento de alcanzar sus propios objetivos personales mientras evita la cooperación para el bien común. Oponiéndose a esta afirmación, Ostrom (2010) considera que, para fomentar la confianza y, por consiguiente, también la cooperación, la reputación y la información personal de cada par deben ser conocidas por todos los miembros de la comunidad. Al conectar el conocimiento a través de comunidades distribuidas y transparentes, los miembros de este tendrán más probabilidades de confiar en otros pares. La suposición de Ostrom (2010) podría corroborarse, por ejemplo, con la tecnología blockchain, que comparte varios principios con la economía colaborativa. El blockchain se basa en una red completamente distribuida donde cada nodo es consciente de las actividades de otros nodos. Altamente simplificado, una transacción entre dos pares no se puede ejecutar a menos que la comunidad verifique y acuerde la viabilidad de la transacción en cuestión. La funcionalidad óptima de las comunidades en línea basadas en redes descentralizadas, como la cadena de bloques, se basa fundamentalmente en la transparencia. Por lo tanto, a diferencia del argumento propuesto por Ostrom (2010), el enfoque de Kaplan y Gurven (2001) perdería peso al explicar la colaboración dentro de redes impulsadas por el conocimiento público, transparente y compartido.

En conclusión, es apropiado llamar la atención sobre el hecho de que la mayoría de los enfoques mencionados en la primera parte de este documento pueden ser cuestionados mediante el análisis de las comunidades globales basadas en plataformas de economía de intercambio en línea. Las grandes redes de cooperación, como Wikipedia o Linux, contradicen la suposición de que los grupos grandes, complejos y heterogéneos son menos propensos a actuar colectivamente (Benkler, 2011; Botsman \& Rogers, 2010). Los participantes activos de tales plataformas sí cooperan, a pesar de que el producto final es libremente compartido y consumido por un mayor número de usuarios pasivos, lo que entra en conflicto con el enfoque de Olson. Además, se ha descubierto que dentro de las comunidades en línea, el parentesco no implica necesariamente la creación de cooperación, a diferencia de 
Martos Carrión, E. De la colaboración fuera de línea a la línea: enfoque teórico sobre los estudios de cooperación humana y la economía colaborativa

conceptos como la confianza y la reputación, que aún desempeñan un papel esencial. Para concluir, la inserción de herramientas digitales en comunidades colaborativas parece haber reinterpretado algunos principios de la cooperación humana. Aún no se ha llevado a cabo una investigación empírica más adecuada y precisa para comprender los nuevos paradigmas de la colaboración en línea, más concretamente los desarrollados dentro del panorama de la economía colaborativa.

\section{AGRADECIMIENTOS}

Este documento de investigación ha sido otorgado por la Charles University Grant Agency (GAUK) y el Science Development Program de la Universidad Charles en Praga (PRVOUK).

\section{REFERENCIAS}

Algar, R. (2007). Collaborative consumption. Leisure Report, 17-18.

Axelrod, R., \& Hamilton, W. (1981). The evolution of cooperation. Science, 211(4489), 13901396.

Bauwens, M. (2012). Synthetic overview of the collaborative economy. Chiang Mai: P2P Foundation.

Benkler, Y. (2004). Sharing nicely: On shareable goods and the emergence of sharing as a modality of economic production. The Yale Law Journal, 114(2), 273.

Benkler, Y. (2011). The penguin and the leviathan; The triumph of cooperation over selfinterest. New York: Crown Business.

Botsman, R., \& Rogers, R. (2010). What's mine is yours: The rise of collaborative consumption. New York, NY: Harper Paperbacks.

Boyd, R., \& Richerson, P. (2005). Solving the puzzle of human cooperation. Evolution and Culture, S. Levinson ed. MIT Press Cambridge MA, 105-132.

Chase, R. (2016). Peers Inc.; How people and platforms are inventing the collaborative economy and reinventing capitalism. London: Headline Book Publishing.

Cosmides, L. \& J. Tooby. (1992). Cognitive adaptations for social exchange. In The Adapted Mind: Evolutionary Psychology and the Generation of Culture (J.H. Barkow, L. Cosmides, J. Tooby, eds.) New York: Oxford University Press, 163-228.

Diani M. \& Eyerman R. (1992). Studying collective action. SAGE Modern Politics series, 30. London: SAGE Publications Ltd.

Felson, M., \& Spaeth, J. (1978). Community Structure and Collaborative Consumption: A Routine Activity Approach. American Behavioral Scientist, 21(4), 614-624.

Gansky, L. (2010). The mesh: Why the Future of Business Is Sharing. New York: Portfolio.

Hardin, G. (1968). The Tragedy of the Commons. Science, 162(3859), 1243-1248.

Howard, B. (2015). We-commerce.; How to Create, Collaborate, and Succeed in the Sharing Economy. USA: Penguin Putnam Inc. 
Martos Carrión, E. De la colaboración fuera de línea a la línea: enfoque teórico sobre los estudios de cooperación humana y la economía colaborativa

Kaplan, H., \& Gurven, M. (2001). The Natural History of Human Food Sharing and Cooperation: A Review and a New Multi-Individual Approach to the Negotiation of Norms. In Conference on the Structure and Evolution of Strong Reciprocity. Santa Fe.

Kendall, D. (2006). Sociology in our times. USA: Thomson Wadsworth.

Lessig, L. (2008). Remix; making art commerce thrive in the hybrid economy. New York: Penguin Books.

Matofska, B. personal interview. (July, 2017). Skype Call from the UK and the Czech Republic.

Olson, M. (1965). The logic of collective action: public goods and the theory of groups. Cambridge, Mass: Harvard University Press.

Ostrom, E. (1990). Governing the commons, The Evolution of Institutions for Collective Action. Cambridge: Cambridge University Press.

Ostrom, E., Poteete, A., \& Janssen, M. (2010). Working Together: Collective Action, the Commons, and Multiple Methods in Practice. Princeton University Press.

Owyang, J. (2013). The Collaborative Economy: Products, services, and market relationships have changed as sharing startups impact business models. To avoid disruption, companies must adopt the Collaborative Economy Value Chain. USA: Altimeter.

Pichardo, N. (1997). New Social Movements: A Critical Review. Annual Review Of Sociology, 23, 411-430.

Rifkin, J. (2014). The zero marginal cost society. New York: Macmillan.

Slee, T. (2015). What is Yours is Mine; The Dark Side of the "Sharing Economy". [S.I.]: OR Books LLC.

Smith, A. (1759). The Theory of moral Sentiments. Andrew Millar, in the Strand; and Alexander Kincaid and J. Bell, Edinburgh

Stephany, A. (2015). The business of sharing; Making it in the newsharing economy. Houndmills, Basingstoke, Hampshire: Palgrave Macmillan in the UK is an imprint of Macmillan Publishers Limited.

Sundararajan, A. (2017). The sharing economy, The End of employment and the rise of crowd-based capitalism. Cambridge, Mass: The MIT Press.

Williams, A., \& Tapscott, D. (2006). Wikinomics, how mass collaboration changes everything. New York: Portfolio.

\section{AUTHOR}

\section{ESTHER MARTOS}

Investigadora en la Universidad Charles de Praga en la República Checa. Su papel dentro del Departamento de Sociología Histórica se centra en la exploración de la economía colaborativa y su impacto social. Estudió ingeniería en diseño industrial, un bachillerato en comunicación y una maestría en artes visuales y multimedia, las tres en la Universidad Politécnica de Valencia en España. Es cofundadora de la plataforma de comida casera p2p Mealby y asesora de economía colaborativa de la plataforma de alquiler de vehículos HireGo, una comunidad totalmente administrada por la tecnología Blockchain. Además, es una de los coordinadores de la Red de Investigación de Economía Colaborativa. 